\title{
Design and Analysis of a THz Metamaterial Structure with High Refractive Index at Two Frequencies
}

\author{
Zan Lu, ${ }^{1}$ Bruno Camps-Raga, ${ }^{2}$ and N. E. Islam ${ }^{1}$ \\ ${ }^{1}$ Electrical and Computer Engineering Department, University of Missouri, Columbia, MO 65201, USA \\ ${ }^{2} \mathrm{MR}$ : comp GmBH, Fuchs Pass 21, 46282 Gelsenkirchen, Germany
}

Correspondence should be addressed to N. E. Islam, islamn@missouri.edu

Received 25 April 2012; Accepted 11 July 2012

Academic Editor: Yasuo Tomita

Copyright () 2012 Zan Lu et al. This is an open access article distributed under the Creative Commons Attribution License, which permits unrestricted use, distribution, and reproduction in any medium, provided the original work is properly cited.

\begin{abstract}
The concept of a single frequency band, single high-refractive-index metamaterial has been extended and applied in the design of dual frequency band, dual high-refractive-index metamaterials in the THz regime. The structure design consists of twenty five unit cells with a surface area of $250 \mathrm{um}$ by $250 \mathrm{um}$ and a thickness of $5 \mathrm{um}$. Each cell has metallic structures embedded in a polyimide substrate. The return loss (S-parameter) analysis shows two strong electric responses at two frequency ranges, and the extracted constitutive parameters suggested high values of simultaneous dielectric constant and permeability at these frequencies. Results retrieved from the S-parameters also show high refractive index values. A first peak refractive index of 61.83 was observed at a resonant frequency of $0.384 \mathrm{THz}$, and another peak refractive index of 19.2 was observed at the resonant frequency $1.416 \mathrm{THz}$. Analysis show that higher refractive index at the second resonance frequency band is achievable through redesign of the structures, and modifications could lead to a single structure with multiple frequency, multiple high-refractive-index metamaterials that can be put to practical use.
\end{abstract}

\section{Introduction}

In recent years, metamaterials have been designed to operate in a broad frequency range, including the microwave [1], terahertz [2], and the infrared [3] regimes. Material design parameters include high refractive index [4], extraordinary optical transmission [5], negative refractive index, permittivity, and permeability values [6-9]. Characterizing the structure responses has been made easier because of the well-known properties of the embedded metallic (e.g., $\mathrm{Cu}$, $\mathrm{Au}$ ) designs. In the $\mathrm{THz}$ regime, Singh and his colleagues have studied the material characteristics extensively [1016], including optically thin metamaterials [10], effects on resonance due to (i) material permittivity [11] and (ii) nearest neighbor interactions [13]. Metamaterials in the $\mathrm{THz}$ regime are of interest because of their applications [17].

In the past few years, great advances in the development of high-refractive-index metamaterials in the $\mathrm{THz}$ regime have been reported. Structures such as metallic wires [18], metallic films with cut slit [19], and specific designed
"I" shape structure [20] have been designed and used in experiments and their performance as high-refractive-index material analyzed.

However, almost all metamaterial structures designed for high-refractive-index do so at a single frequency band. Since potential applications in the $\mathrm{THz}$ regime could require wideband, it would be desirable, for practical usage, to have a multiband high refractive index metamaterials. Thus, the focus of research for many is the design of multi- $\mathrm{THz}$, high-refractive-index metamaterials. Design of two resonant frequencies in a metamaterial structure has been reported earlier [21, 22], but the high refractive index needed for the applications was not shown. In this paper, we report on the development of a metamaterial structure that can have two or more wide bands with high-refractive-index value in the $\mathrm{THz}$ regime. With this brief introduction, the rest of the paper is divided as follows: in Section 2, the theory on designing the structure is described, while Section 3 shows the simulation and the design effort. The results are analyzed in Section 4, while we conclude in Section 5. 


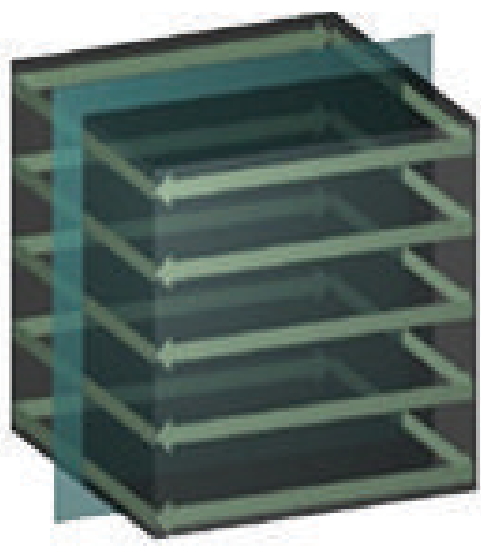

(a)

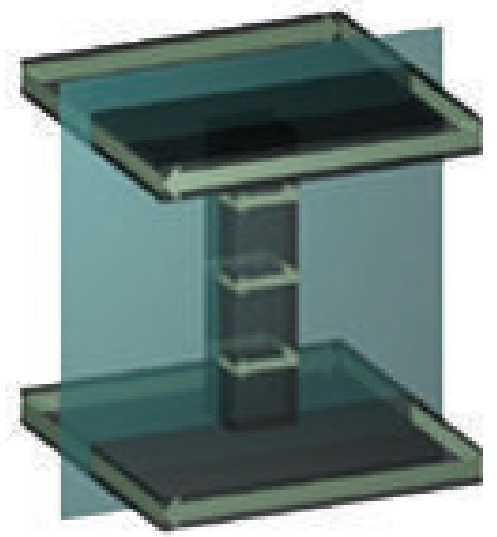

(b)

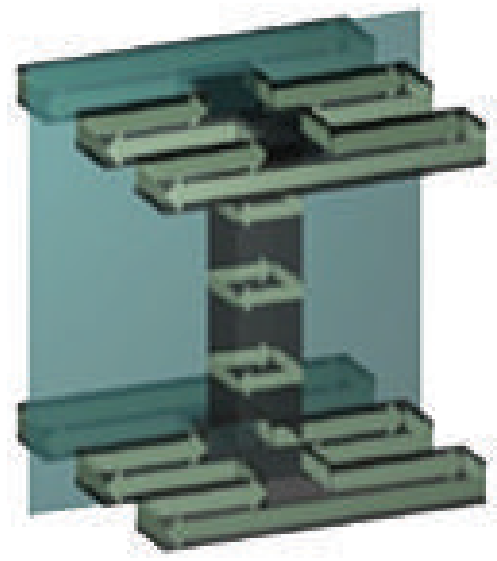

(c)

FIgUre 1: Current distribution when magnetic field is applied to the structure, from [23].

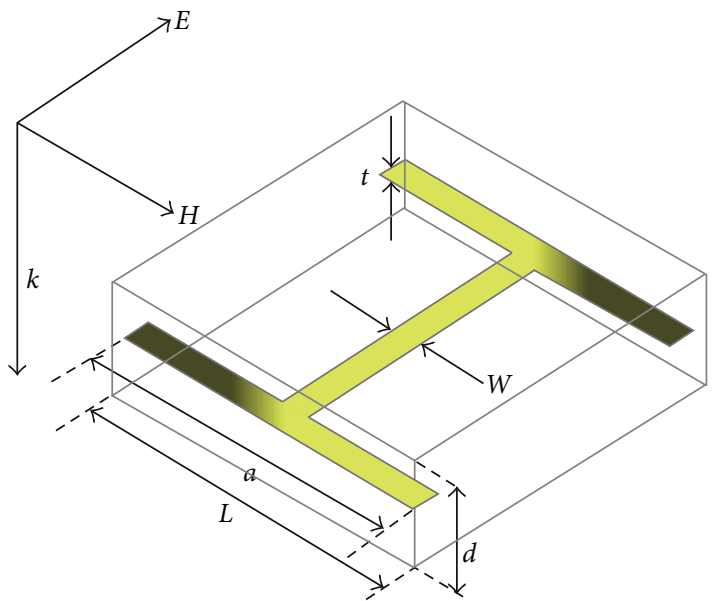

Figure 2: Schematic plot of the single "I" shape structure, from $[20]$.

\section{Background}

The refractive index $n$ is related to a material's relative permittivity and the relative permeability $\varepsilon_{r}$ and $\mu_{r}$, through the relationship $n=\sqrt{\varepsilon_{r} \times \mu_{r}}$. To achieve a higher-refractiveindex value in a metamaterial design, the dielectric constant and the permeability need to be enhanced at the same time. A high dielectric constant value can be achieved through an electric resonance that results from a strong capacitive response, as indicated in aprevious work [18, 19]. However, such designs may also have a strong diamagnetic response induced by the current loop on the surface of the structure that is normal to the electric field, thus making the effective permeability of the metamaterial relatively small $(u \ll 1)$. A reduction of the diamagnetic effect in the structure can be achieved through interactions with a propagating electromagnetic wave, by reducing the area subtended by the current loop that is parallel to the magnetic field to decrease the magnetic dipole moment to the maximum

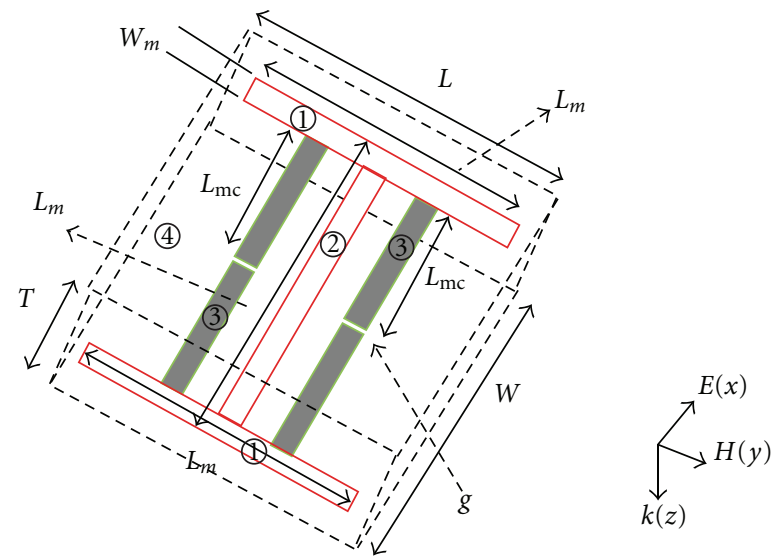

Figure 3: Schematic plot of the single-unit cell design.

extent possible. In this way, the strong capacitive response is maintained while the diamagnetic response is suppressed in the structure. Shin and his research utilized the principle of diamagnetic response suppression in producing a highrefractive-index value [23].

Figures $1(\mathrm{a})-1(\mathrm{c})$ show the configuration used by Shin in suppressing the diamagnetic effect of the structure. Figure 1(a) shows a metallic cube-like structure exposed to the magnetic field with a large area surrounded by the current loops induced on the surface of the structure, while Figure 1(b) shows the modified structure with a bar connecting the top and bottom panel to decrease the area surrounded by the induced current loop. In Figure 1(c), it shows an even improved structure with cut slits on the top and bottom panel of the structure to reduce the area surrounded by the current loop while maintaining the electric charge. According to the fringe-field effect [24, 25], the total surface charge on the top and bottom panel remains the same as the one without cut slits. As a result, the electric response remains the same while the diamagnetic response 


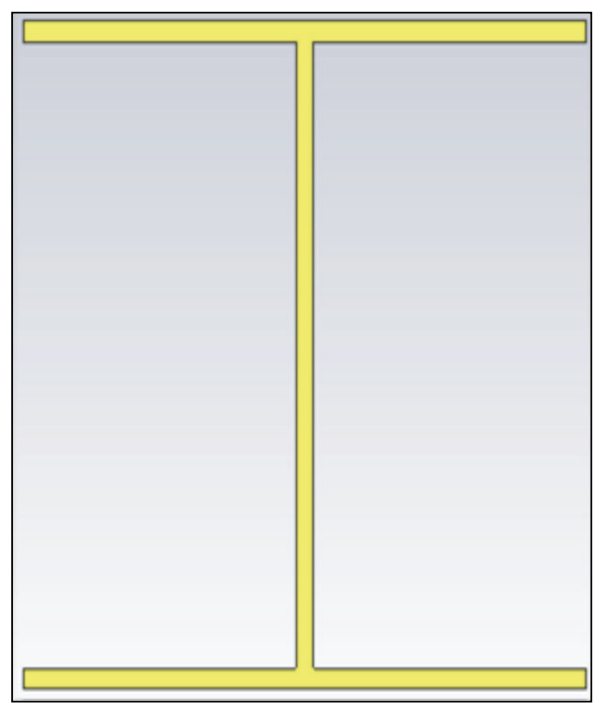

(a)

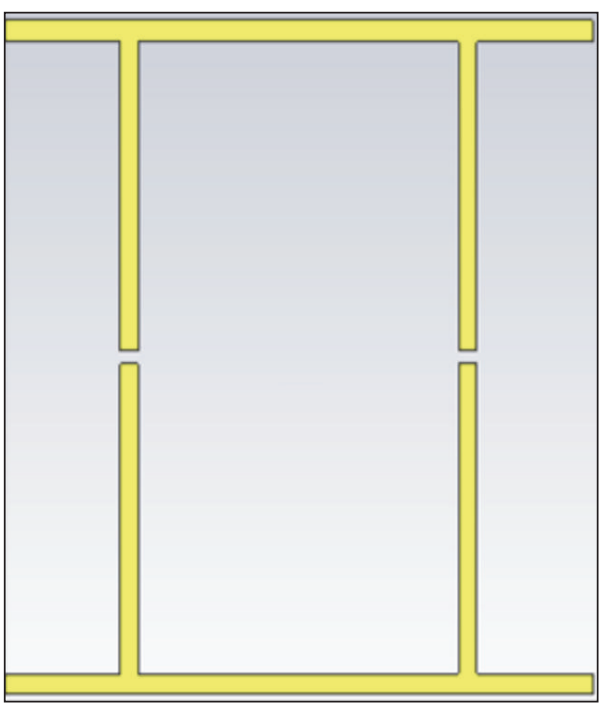

(b)

FIGURE 4: Schematic plot of the single band structures. (a) is the "I" shape structure with a lower resonant frequency band, and (b) is the cut wire structure with a higher resonant frequency band.

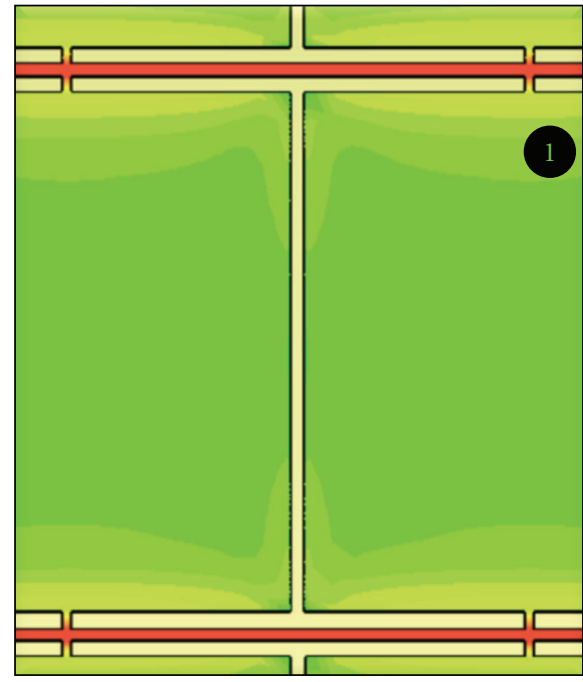

(a)

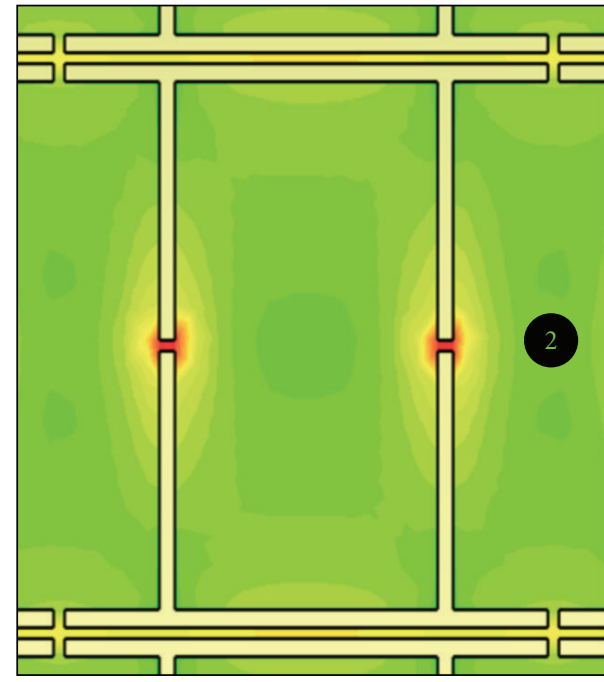

(b)

FIGURE 5: Normal of the electric field distribution on the single band structures. (a) is the field distribution on the "I" shape structure, and (b) is the field distribution on the cut wire structure.

is reduced when the area parallel to the magnetic field as produced in Figure 1(a) is made smaller by etching the surfaces as shown in Figure 1(c).

Another "I" shape structure in the terahertz frequency range that shows ultrahigh electric resonant value and low diamagnetic response was introduced by Choi recently [20] and is shown in Figure 2. This structure has high refractive index optimized from Shin's work [23].

However, all such structures designed so far exhibit high refractive index at a single $\mathrm{THz}$ frequency band. Thus, there is a need to design a dual or multiband metamaterial structure with high refractive index at each band, such that a single structure can be tuned to a specific application, if necessary. In this paper, we design and discuss such a metamaterial structure that has multiband, high-refractiveindex values [26]. Our research builds on and integrates previous work discussed earlier.

\section{Design and Simulation}

Figure 3 shows the unit structure of the proposed metamaterial that produces high refractive index at each frequency band. Like the previously described single-band highrefractive-index material, this structure is also the result of 


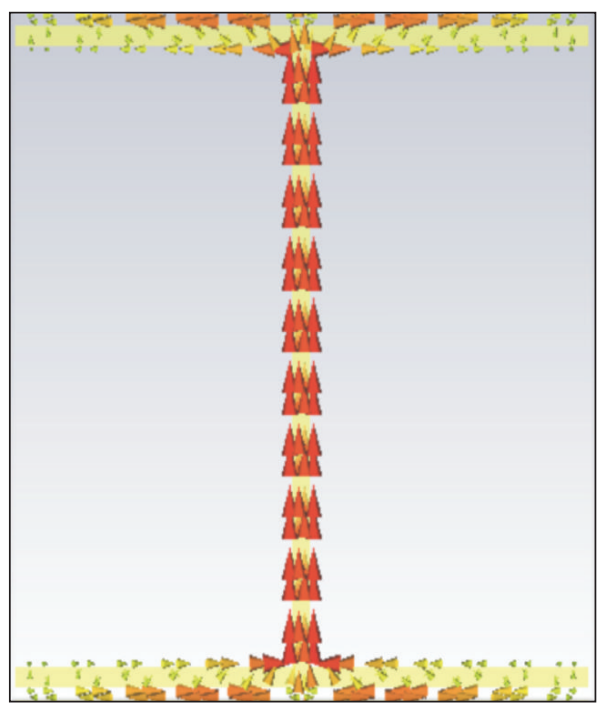

(a)

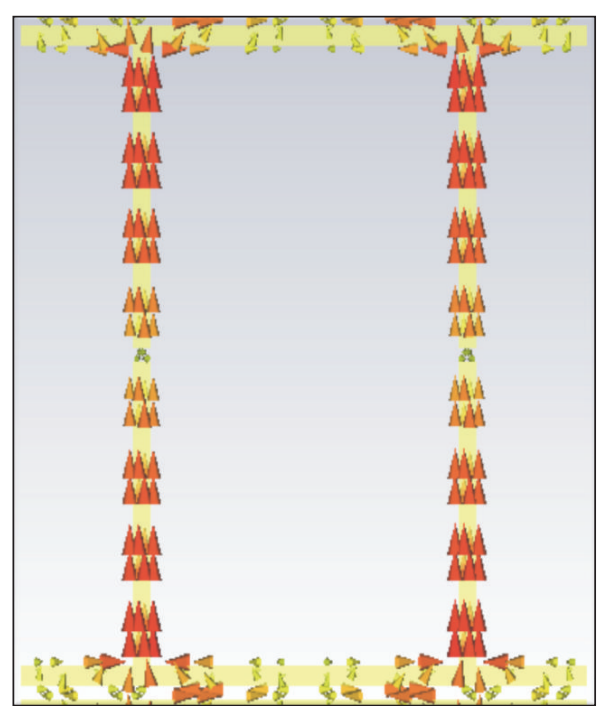

(b)

FIGURE 6: Surface current distribution of the single band structures. (a) shows the current distribution of the single "I" shape structure, and (b) shows the current distribution of the cut wire structures.

TABLE 1: Dimensions and components of the designed structure.

\begin{tabular}{lccc}
\hline Location & Length & Width & Thickness \\
\hline (1) Top and bottom bar, $\mathrm{Au}$ & $L_{m}=49 \mathrm{um}$ & $W_{m}=1.5 \mathrm{um}$ & $0.1 \mathrm{um}$ \\
(2) Center bar, $\mathrm{Au}$ & $L_{m}-2 \times W_{m}=46 \mathrm{um}$ & $W_{m}=1.5 \mathrm{um}$ & $0.1 \mathrm{um}$ \\
(3) Cut wires, $\mathrm{Au}$ & $g=L-W ; L_{m c}=(46-g) / 2$ & $W_{m}=1.5 \mathrm{um}$ & $0.1 \mathrm{um}$ \\
(4) Substrate, polyimide & $L=50 \mathrm{um}$ & $W=49 \mathrm{um}$ & $5 \mathrm{um}$ \\
\hline
\end{tabular}

a number of iterations in the simulation effort that finally resulted in the configuration shown here.

The metallic structure (gold) is embedded in the center of a substrate made with polyimide. The thickness of the embedded metallic structure is $0.1 \mathrm{um}$, and the width of the metal is kept at $1.5 \mathrm{um}$ in order to reduce the diamagnetic effect caused by the magnetic dipole that results from the current loop surrounding the structure. Table 1 details the dimensions associated with of the unit cell shown in Figure 3. Commercial software, the CST Microwave Studio, was used to simulate the effects of electromagnetic wave interactions with the metamaterial. The background and boundary conditions of the unit cell were set up properly to make the wave propagate and polarize in the desired direction. In the simulation, two waveguide ports were used to generate and receive the polarized $\mathrm{THz}$ wave, which is incident normally on the substrate along the $z$-direction with the electric field in the $x$-direction parallel to the metallic structure and magnetic field in the $y$-direction. It should be noted that the properties of the $\mathrm{THz}$ material could be affected at oblique incidence [24] and are not studied here.

3.1. Individual Structure Simulation. Since the modified design for dual frequency response presented here combines two separate high-refractive-index single frequency metamaterial designs, the response mechanism of the modified design can be best understood when the single frequency structures are analyzed first. Figures 4(a) and 4(b) show the two high refractive index, single frequency structures. In both these designs, the metallic structures are embedded in polyimide foam and have five by five unit cells. The dimensions of both the structures are kept the same so that a reasonable comparison of their characteristics is possible.

When exposed to the electromagnetic field, surface charges accumulate on the top and bottom bar in the $y$ direction, which will then induce surface current along the center bar and the cut wires, as shown in Figures 6(a) and 6(b) and capacitors developed between the units in structure of Figure 5(a), while similar capacitors developed between the gaps of the two split bars in Figure 5(b). These capacitive resonances are reflected as two electric responses. Figures 5(a) and 5(b) show the induced electric resonance areas, marked as in Figure 5(a) and in Figure 5(b) with red color indicating the highest level of the induced electric field. This will be evident in the transmission plot and the extracted permittivity and permeability plot later.

These electric responses are also evident from the surface current distribution on the metallic structure. Figure 6(a) shows a strong current induced along the centers bar of the structure, resulting in the capacitive response between the gaps which induces the electric resonances. On the other hand, Figure 6(b) shows two induced surface current on the two sets of cut wires along the same direction. The two capacitive responses in the gaps couple with each other to 


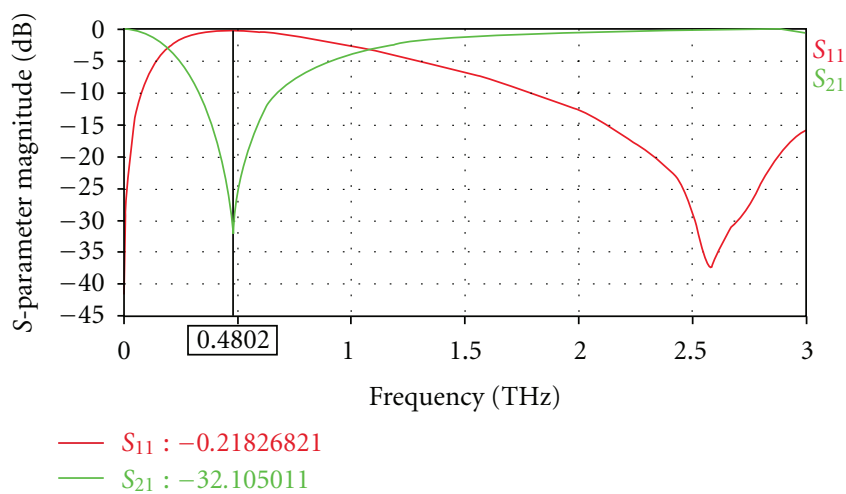

FIGURE 7: Transmission and reflection of the "I" shape structure. The resonant frequency is around $0.48 \mathrm{THz}$.

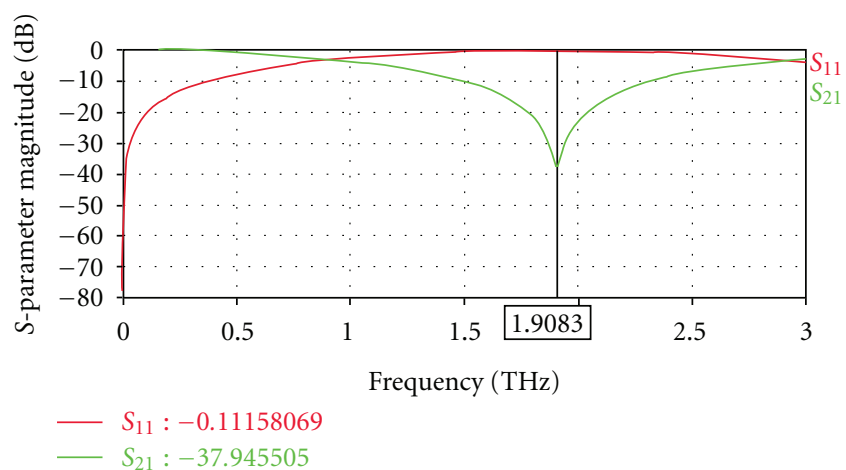

FIgURE 8: Transmission and reflection of the cut wire structure. The resonant frequency is around 1.9 THz.

provide a strong electric resonance, which is even stronger than the single-bar resonance. The resonance of the two structures is displayed in the plot of the transmittance in Figure 7. The lowest value of the transmission indicates the resonant frequency of the structure.

The resonance frequency band for structures in Figures 5(a) and 5(b) can be determined from the transmittance and reflectance plots of the structures, as shown in Figures 7 and 8. For the structure in Figure 5(a), the resonant frequency lies near $0.48 \mathrm{THz}$ where the transmission reaches $-32 \mathrm{~dB}$, while structure in Figure 5(b) has a resonant frequency at near $1.9 \mathrm{THz}$ where transmission reaches $-38 \mathrm{~dB}$. The second resonant frequency exhibits lower and broader electric response than the first one (Figure 8). We consider that the current distribution on the two sets of split cut wires and the corresponding capacitive response contribute the most to the second resonant frequency of the structure. The difference between the two plots indicates that it might have more broad use within the second resonant frequency band, which is even broader than the first resonant frequency band.

3.2. Combined Structure Simulation. The combined structure is shown in Figure 9, where the dimensions are the same as the single band structure simulated in the previous section.

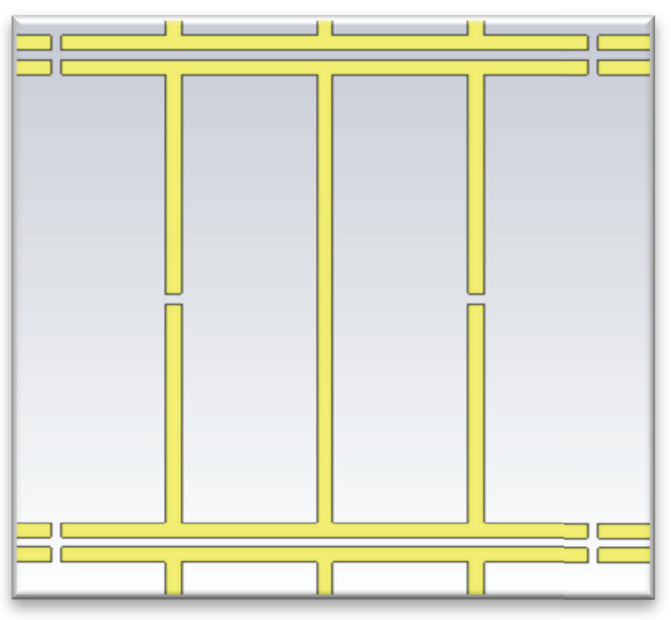

FIGURE 9: Schematic plot of the new design with dual band of resonant frequency.

Figure 10 is the transmittance and reflectance of the simulated combined structure. As known, the lowest point of the two transmissions reflects the strongest point of the resonance. The result clearly exhibits two strong resonant frequencies with a lower value of $0.416 \mathrm{THz}$ and another one at a higher frequency of $2.046 \mathrm{THz}$. 


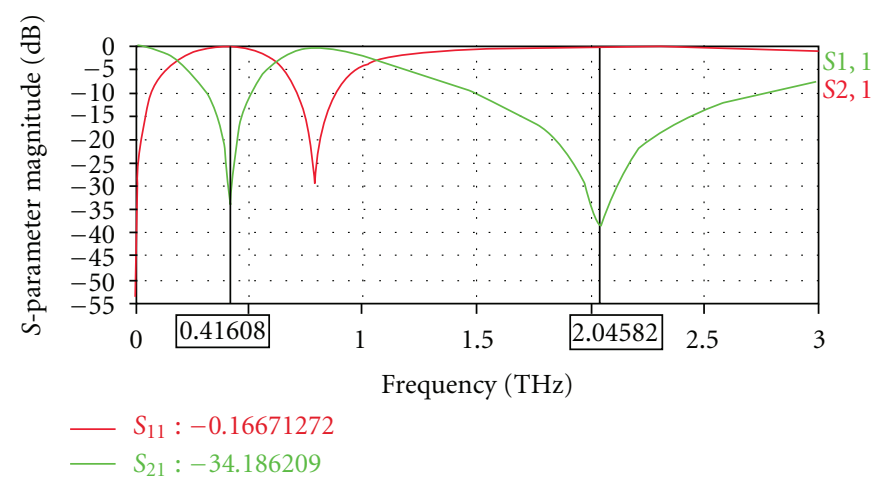

Figure 10: Transmission and reflection of the designed dual band metamaterial.

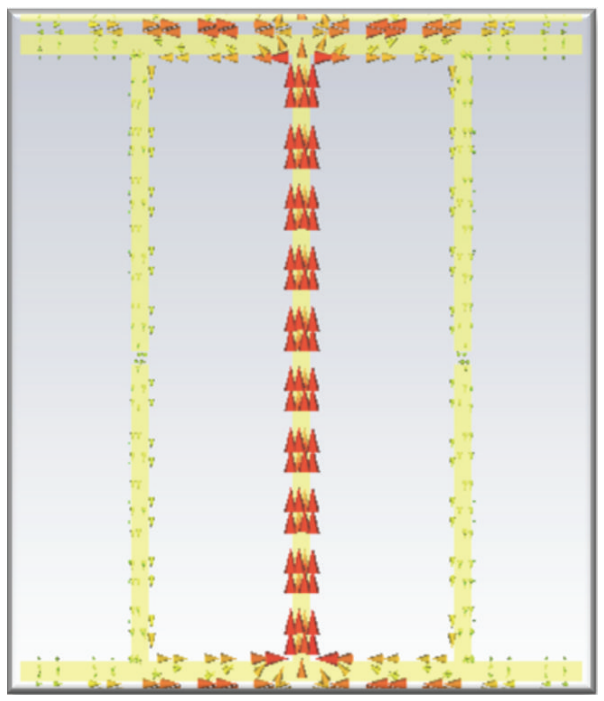

(a)

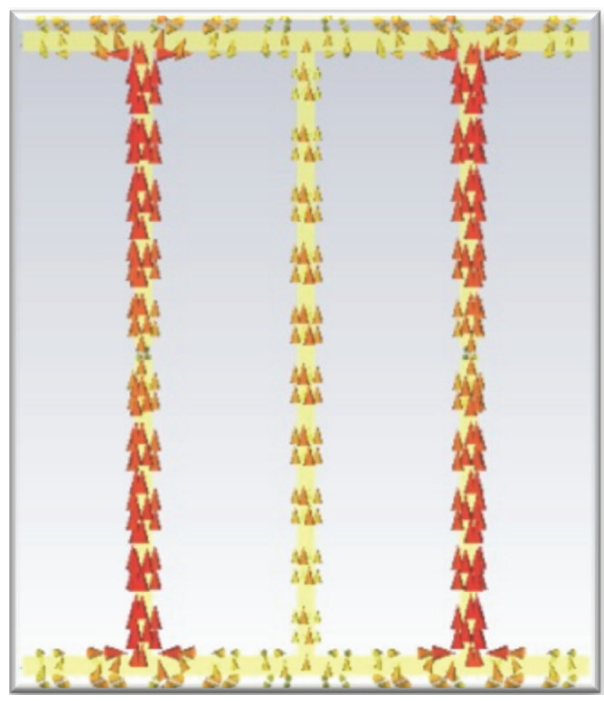

(b)

Figure 11: Current distribution at different resonant frequencies. The left figure indicates the lower resonant frequency with strong current distribution in the center bar. The figure on the right indicates the higher resonant frequency with current distribution mostly on the cut wires.

Here, the resonant frequencies of the combined structure have shifted a little as compared to the two individual structures shown earlier. The shift in the resonant peak frequency could be attributed to the coupling of the two surface currents induced in the modified structure at two resonance frequencies.

The structure in Figure 11(a) shows the current distribution at a resonant frequency of $0.416 \mathrm{THz}$. In this case, the electric response is mainly due to the strong current induced in the center bar. The structure in Figure 11(b) shows a current distribution at a higher resonance frequency of $2.046 \mathrm{THz}$. Here, the majority of the current is distributed on the side cut wires. These current distribution plots at different frequencies clearly show not only onset of two resonance frequencies for the modified structure, but also the fact that due to the presence of charges in the structures, there is a shift in the resonance frequencies when the original structure had only one embedded metallic structure in the dielectric. The final step in our analysis is to demonstrate the high-refractive-index values in the two resonant frequencies for the proposed modified structure. We only compare the two cases: the "I" shape (Figure 5(a)) structure and the new modified structure as shown in Figure 9.

\section{High-Refractive-Index Analysis}

The intrinsic properties of the metamaterial structures can be retrieved by using the scattering parameters S11 and S21 and a method previously used by Smith and so forth [26]. The effective permittivity and the effective permeability for the comparison in both cases are extracted from the Sparameters, which are shown in the plot as the transmission and reflection of the simulated structure. Figures 7 and 10 show the S-parameter for the single frequency band structure (Figure 5(a)) and the dual frequency band modified structure (Figure 9), respectively.

In the first case of the "I" shape structure, a strong electric response and a high value of relative permeability are evident due to a strong coupling between the metallic 

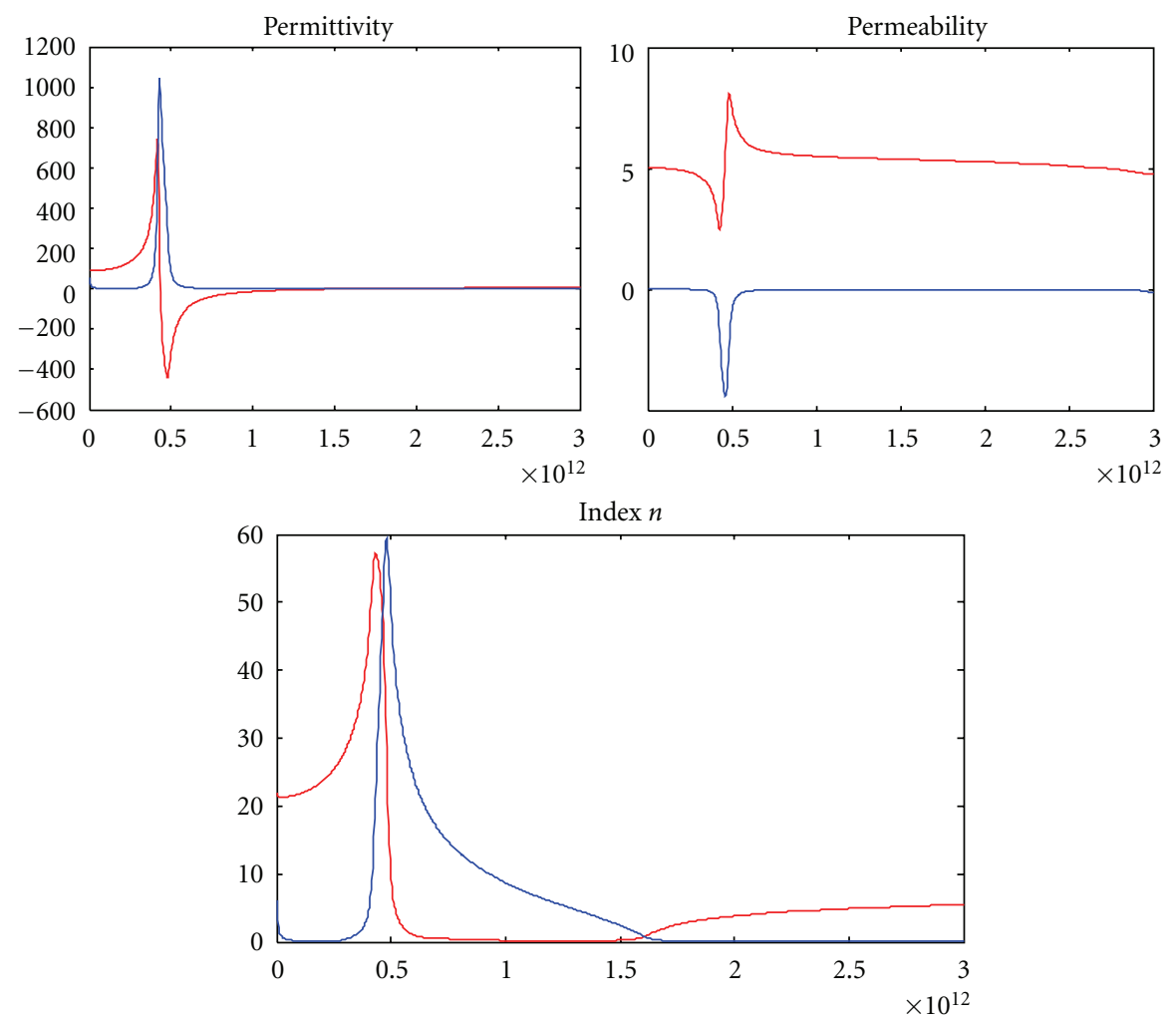

FIGURE 12: The upper left and right are the permittivity and permeability of the single frequency band metamaterial. The lower figure is the extracted refractive index of the metamaterial.
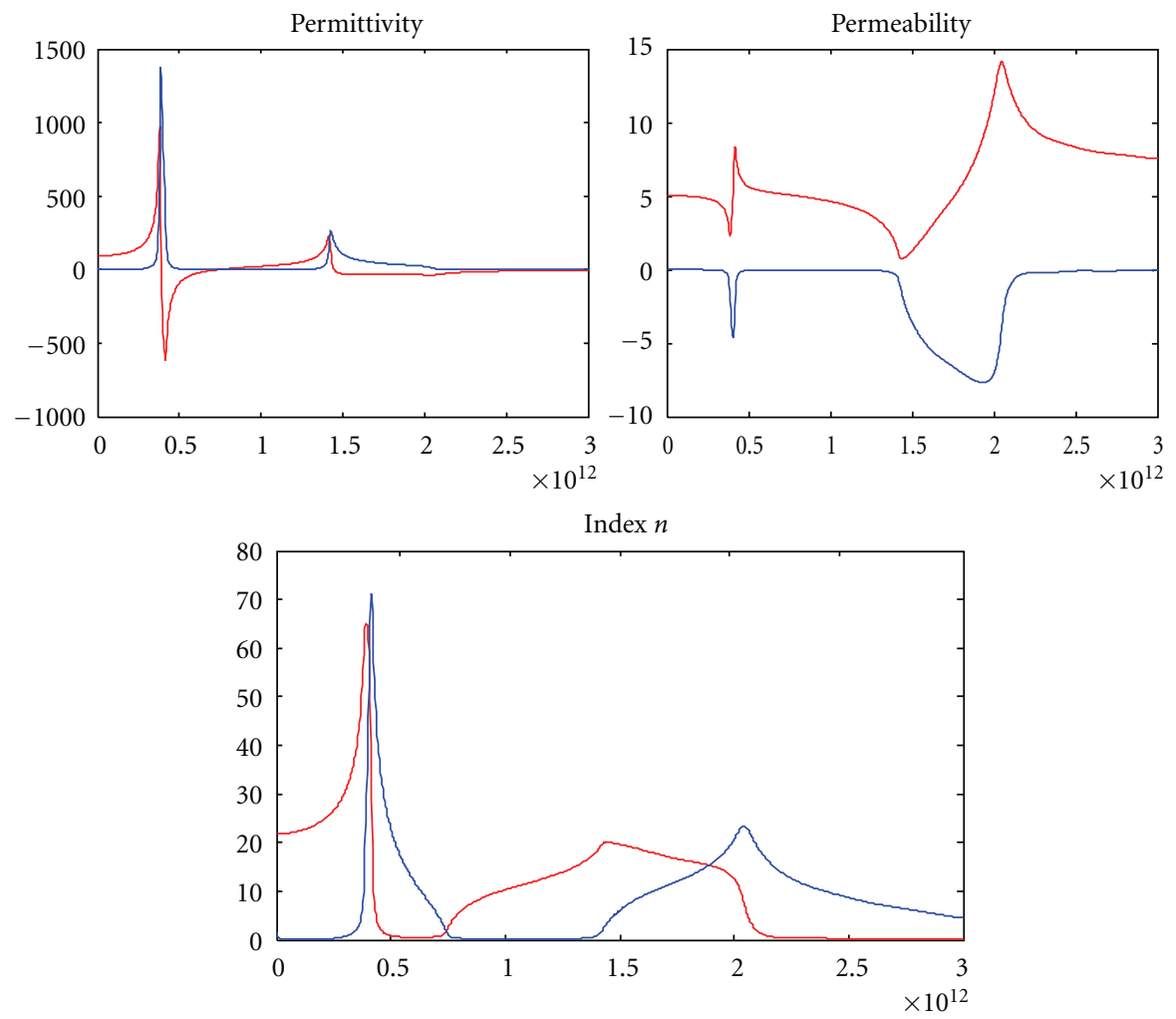

FIGURE 13: The upper left and right are the permittivity and permeability of the dual frequency band metamaterial. The lower figure is the extracted refractive index of the metamaterial. 
units and the small area surrounded by the current loop parallel to the magnetic field. Figure 12 shows the retrieved permittivity and permeability of the "I" shape structure, where the relative permittivity reaches a high value of 715.1 at the frequency of $0.42 \mathrm{THz}$, and the relative permeability is 2.646 at the same frequency. The corresponding refractive index reaches 53.42 at this frequency. The result on one hand approves the research and theory of the early design [19], while on the other hand, it provides the comparison with the dual resonant structure in the resonant frequency band and the refractive index value.

Compared with the single frequency band structure in the first case, there exists, significant difference in the resonant frequency and the intrinsic parameters in the second case of the dual band frequency structure. As seen in Figure 13, there are two resonance peaks in the operating frequency range which results in two relative permittivity peak values of the 802.6 and 227 at $0.384 \mathrm{THz}$ and $1.416 \mathrm{THz}$, respectively. The corresponding relative permeability at these frequencies is 2.381 and 1.153 . Thus, the refractive index at $0.384 \mathrm{THz}$ is 61.83 and 19.2 at $1.416 \mathrm{THz}$.

The first resonant frequency proves an enhancement in the relative permittivity due to the coupling effects between the center bar and the cut wires and a small decrease in the relative permeability due to the enlarged area of surface surrounded by the current loops. However, the second electric and magnetic resonances in the operating frequency appear to be not as strong as the first one in this design. The reason for the weak second resonant frequency band could be due to the fact that at this higher frequency a homogenous medium approximation would fail and thus would result in the elimination of the same direction-induced surface current on the cut wire. Since the second resonant frequency as we see is mainly decided by the cut wires, the same direction current loop on the cut wires eliminates the electric and magnetic response and results in a smaller capacitive resonance and a smaller relative permeability compared to those with the first resonant frequency.

As is evident from Figure 13, the second refractive index value is in the broadband range. Since the property of the metamaterial depends mostly on the geometry and the arrangement of the structure, we believe that the dimensions of the structure can be modified to get a stronger resonance at the desired frequency.

Finally, as discussed earlier in Section 2 and extensively by Shin et al. [23], the structure design suppresses the strong diamagnetic response of the structure, and extracted, effective permeability for the structure is more than the expected value, since inhomogeneity of the structure was not considered for parameter extraction [27], and the figures do not represent the exact profile.

\section{Conclusion}

A high-refractive-index metamaterial structure with dual frequency band was designed through simulation and analysis. Results show that the metamaterial, designed with gold structures embedded with polyimide film, effectively exhibits a high refractive index at two frequency bands.
In the second frequency band, the high refractive index is of relatively smaller value than the main effective band. It is expected that through modifications in geometry and design, improvement in the electric and magnetic resonant frequency and the refractive index value can be enhanced for the second frequency band.

The methodology applied in this study could be applied to structures to design high-refractive-index metamaterials at multifrequency. Since polyimide has good flexibility and isolation property, the designed structure can be used in a variety of applications.

\section{References}

[1] M. C. K. Wiltshire, J. B. Pendry, I. R. Young, D. J. Larkman, D. J. Gilderdale, and J. V. Hajnal, "Microstructured magnetic materials for rf flux guides in magnetic resonance imaging," Science, vol. 291, no. 5505, pp. 849-851, 2001.

[2] T. J. Yen, W. J. Padilla, N. Fang et al., "Terahertz magnetic response from artificial materials," Science, vol. 303, no. 5663, pp. 1494-1496, 2004.

[3] S. Zhang, W. Fan, N. C. Panoiu, K. J. Malloy, R. M. Osgood, and S. R. J. Brueck, "Experimental demonstration of near-infrared negative-index metamaterials," Physical Review Letters, vol. 95, no. 13, Article ID 137404, 4 pages, 2005.

[4] J. T. Shen, P. B. Catrysse, and S. Fan, "Mechanism for designing metallic metamaterials with a high index of refraction," Physical Review Letters, vol. 94, no. 19, Article ID 197401, 4 pages, 2005.

[5] T. W. Ebbesen, H. J. Lezec, H. F. Ghaemi, T. Thio, and P. A. Wolff, "Extraordinary optical transmission through sub-wavelenght hole arrays," Nature, vol. 391, no. 6668, pp. 667-669, 1998.

[6] J. B. Pendry, A. J. Holden, D. J. Robbins, and W. J. Stewart, "Magnetism from conductors and enhanced nonlinear phenomena," IEEE Transactions on Microwave Theory and Techniques, vol. 47, no. 11, pp. 2075-2084, 1999.

[7] D. R. Smith and N. Kroll, "Negative refractive index in lefthanded materials," Physical Review Letters, vol. 85, no. 14, pp. 2933-2936, 2000.

[8] S. O’Brien and J. B. Pendry, "Magnetic activity at infrared frequencies in structured metallic photonic crystals," Journal of Physics Condensed Matter, vol. 14, no. 25, pp. 6383-6394, 2002.

[9] F. J. Rachford, D. L. Smith, P. F. Loschialpo, and D. W. Forester, "Calculations and measurements of wire and/or split-ring negative index media," Physical Review E, vol. 66, no. 3, Article ID 036613, 5 pages, 2002.

[10] R. Singh, E. Smirnova, A. J. Taylor, J. F. O’Hara, and W. Zhang, "Optically thin terahertz metamaterials," Optics Express, vol. 16, no. 9, pp. 6537-6543, 2008.

[11] R. Singh, A. K. Azad, J. F. O'Hara, A. J. Taylor, and W. Zhang, "Effect of metal permittivity on resonant properties of terahertz metamaterials," Optics Letters, vol. 33, no. 13, pp. 15061508, 2008.

[12] R. Singh, E. Plum, C. Menzel et al., "Terahertz metamaterial with asymmetric transmission," Physical Review B, vol. 80, no. 15, Article ID 153104, 4 pages, 2009.

[13] R. Singh, C. Rockstuhl, F. Lederer, and W. Zhang, "The impact of nearest neighbor interaction on the resonances in terahertz metamaterials," Applied Physics Letters, vol. 94, no. 2, Article ID 021116, 3 pages, 2009. 
[14] R. Singh, A. A. I. Al-Naib, M. Koch, and W. Zhang, "Sharp Fano resonances in THz metamaterials," Optics Express, vol. 19, no. 7, pp. 6312-6319, 2011.

[15] R. Singh, X. Lu, J. Gu, Z. Tian, and W. Zhang, "Random terahertz metamaterials," Journal of Optics A, vol. 12, no. 1, Article ID 015101, 2010.

[16] R. Singh, E. Plum, W. Zhang, and N. I. Zheludev, "Highly tunable optical activity in planar achiral terahertz metamaterials," Optics Express, vol. 18, no. 13, pp. 13425-13430, 2010.

[17] Z. Shi and W. Robert Boyd, "Slow-light Fourier transform interferometer," Physical Review Letters, vol. 99, no. 24, Article ID 240801, 4 pages, 2007.

[18] X. Wei, H. Shi, X. Dong, Y. Lu, and C. Du, "A high refractive index metamaterial at visible frequencies formed by stacked cut-wire plasmonic structures," Applied Physics Letters, vol. 97, no. 1, Article ID 011904, 3 pages, 2010.

[19] A. Pimenov and A. Loidl, "Experimental demonstration of artificial dielectrics with a high index of refraction," Physical Review B, vol. 74, no. 19, Article ID 193102, 3 pages, 2006.

[20] M. Choi, S. H. Lee, Y. Kim et al., "A terahertz metamaterial with unnaturally high refractive index," Nature, vol. 470, no. 7334, pp. 369-373, 2011.

[21] Y. Yuan, C. Bingham, T. Tyler et al., "A dual-resonant terahertz metamaterial based on single-particle electric-field-coupled resonators," Applied Physics Letters, vol. 93, no. 19, Article ID 191110, 3 pages, 2008.

[22] Y. Yuan, C. Bingham, T. Tyler et al., "Dual-band planar electric metamaterial in the terahertz regime," Optics Express, vol. 16, no. 13, pp. 9746-9752, 2008.

[23] J. Shin, J. T. Shen, and S. Fan, “Three-dimensional metamaterials with an ultrahigh effective refractive index over a broad bandwidth," Physical Review Letters, vol. 102, no. 9, Article ID 093903, 4 pages, 2009.

[24] C. Menzel, R. Singh, C. Rockstuhl, W. Zhang, and F. Lederer, "Effective properties of terahertz double split-ring resonators at oblique incidence," Journal of the Optical Society of America B, vol. 26, no. 12, pp. B143-B147, 2009.

[25] Z. Lu, B. Camps-Ragas, and N. E. Islam, "Design and analysis of multiband high refractive index metamaterials," in IEEE International Symposium on Antennas and Propagation and USNC-URSI National Radio Science Meeting, IEEE Conference Proceedings, pp. 8-14, Chicago, Ill, USA, July 2012.

[26] D. R. Smith, S. Schultz, P. Markoš, and C. M. Soukoulis, "Determination of effective permittivity and permeability of metamaterials from reflection and transmission coefficients," Physical Review B, vol. 65, no. 19, Article ID 195104, 5 pages, 2002.

[27] D. R. Smith, D. C. Vier, T. Koschny, and C. M. Soukoulis, "Electromagnetic parameter retrieval from inhomogeneous metamaterials," Physical Review E, vol. 71, no. 3, Article ID 036617, 11 pages, 2005. 

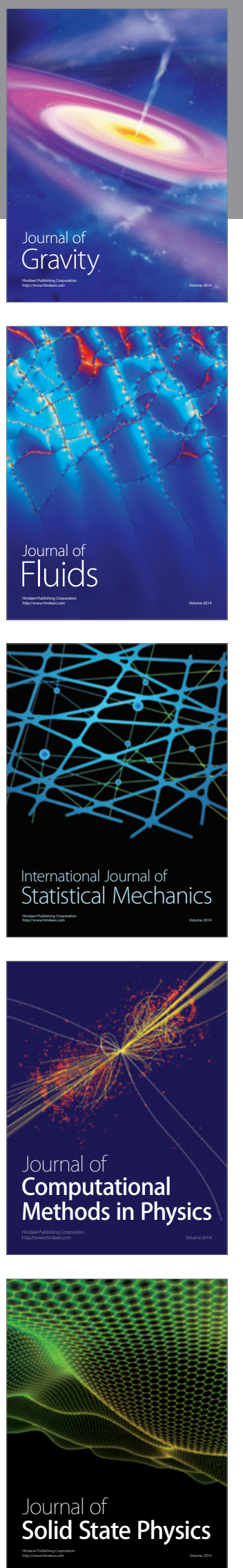

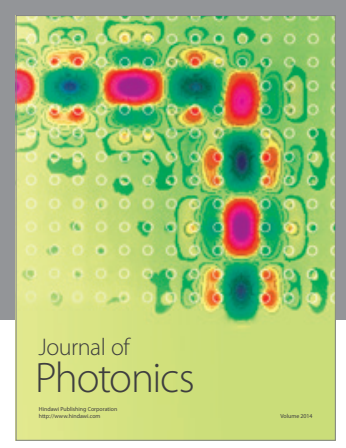

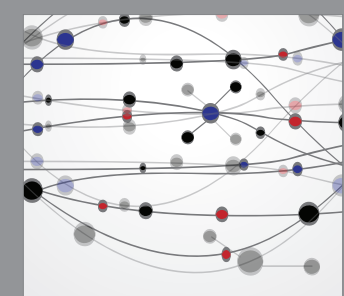

The Scientific World Journal
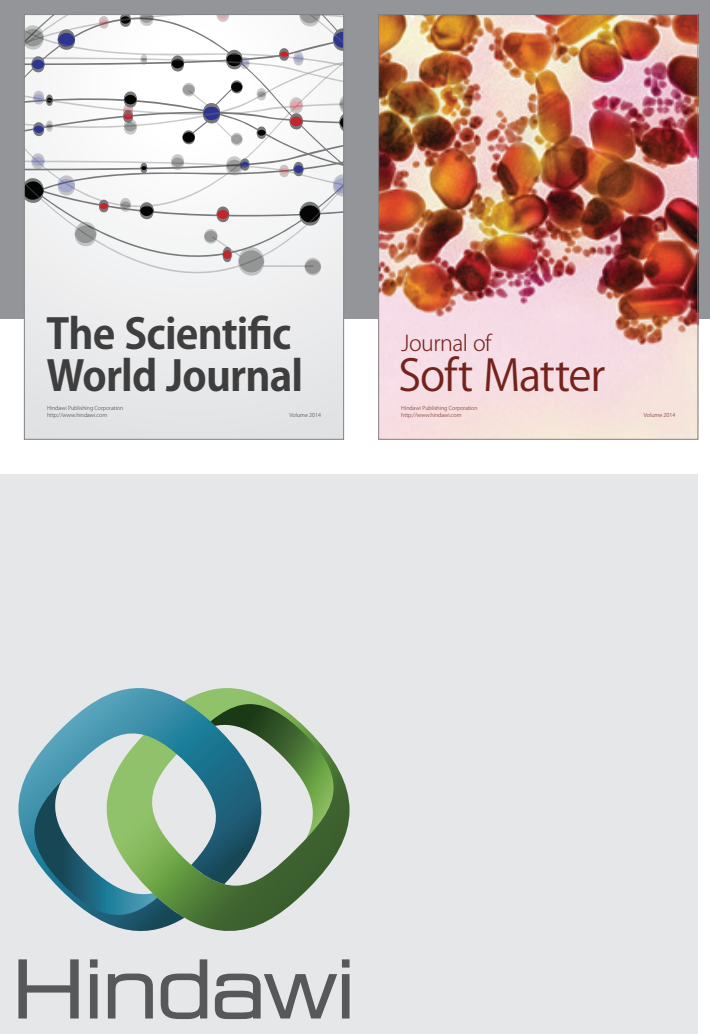

Submit your manuscripts at

http://www.hindawi.com
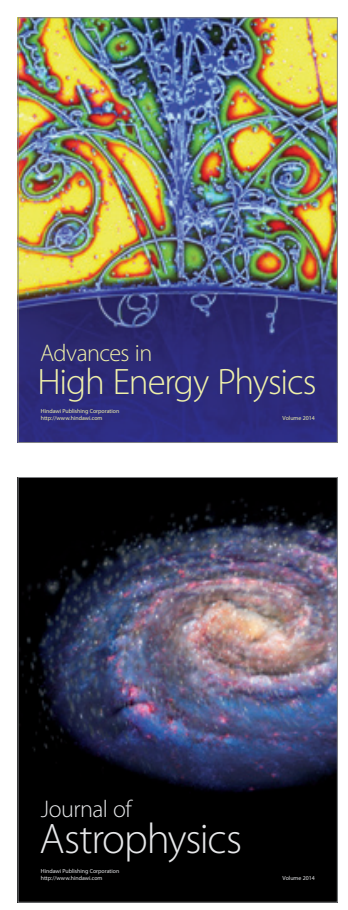
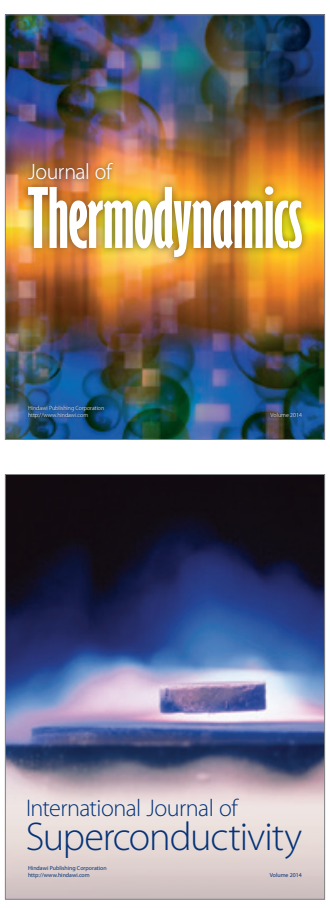
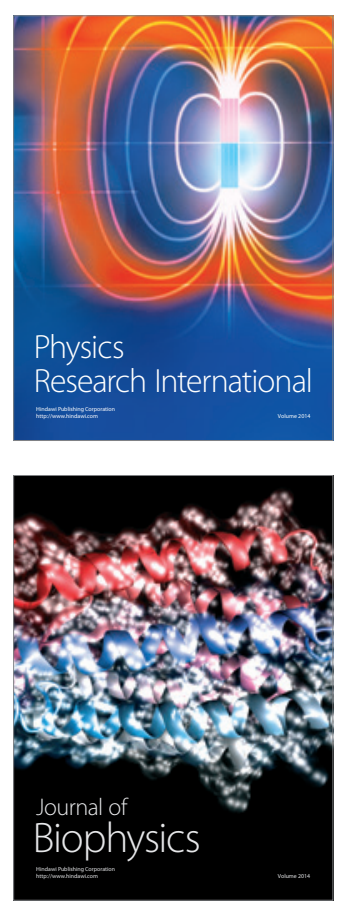
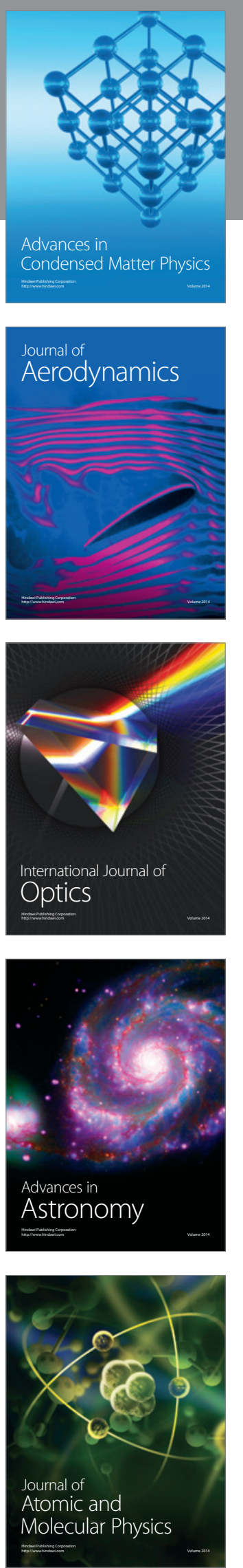